\title{
Zona Incerta Stimulation Likely to be Particularly Effective in Central Post- \\ Stroke Pain.
}

Paul D. McGeoch MD MRCP FRCS(SN)

Dept of Medicine,

University of Bristol,

U.K.

Dept of Medicine,

Queen's University,

Belfast,

U.K.

Lu et al. (2021) recently reported that deep brain stimulation (DBS) of the zona incerta (ZI) selectively modulates thermal pain in humans.[1] This important finding has implications for the mechanism and treatment of neuropathic pain and particularly, as I explore in this paper, central post-stroke pain (CPSP). The condition, now termed CPSP, was first described in 1906 by Dejerine and Roussy, who noted that "nothing offers solace to the patient whose feeling are sometimes intolerable".[2] Over a century later CPSP remains a difficult, often refractory, condition to treat.[3] One that can even drive sufferers to suicide.[4]

Lu et al. studied 11 patients who had DBS electrodes placed, for the treatment of Parkinson's disease, in the region of the ZI, which is in the subthalamic area. They found that stimulating these electrodes at a frequency of $20 \mathrm{~Hz}$ could contralaterally reduce experimental heat pain by a significant amount. Conversely, no alterations in warmth perception or pressure pain were observed. The authors noted that the DBS leads were in the dorsolateral ZI whereas the portions of ZI connected to the spinothalamic tract and 
sensory thalamus are more ventromedial. They went on to suggest that directly targeting the ventral ZI could improve the consistency and magnitude of the observed analgesic effect. Lu et al.'s report is valuable and potentially opens a new therapeutic avenue in the treatment of neuropathic pain. However, its discussion section could be usefully expanded in three important and related ways, as outlined below.

First, a consideration of the neural pathways involved in interoception and homeostasis would help inform on the likely underlying neurological mechanisms involved. There is a lot of evidence that it is not the sensorimotor cortex that is the main cortical projection targets of the lamina I spinothalamic tract (STT).[5-7] Rather the main STT projection targets in the cortex are the dorsal posterior insula (dpIns) and the anterior cingulate cortex (ACC).[5-7] (There is also a lesser projection to the fundus of the central sulcus between the sensory and motor cortices).[6]

The dpIns can be viewed as interoceptive cortex, representing the current physiological state of the body, and is associated with the discriminative component of pain - i.e. what it feels like. Conversely, the ACC is involved in motivation and the affective aspect of pain - i.e. the desire to do something about it. $[5,6]$ The ACC and dpIns are important cortical homeostatic sites and both are active during the perception of thermal pain. $[6,8,9]$ Thus, if ZI DBS is modulating thermal pain it is likely modulating one, or both, of these cortical areas.

Second, following on from this, that ZI DBS specifically ameliorates thermal pain suggests that it may be particularly effective in treating CPSP. This is because impaired temperature perception is a near ubiquitous finding in CPSP patients.[10] Indeed, a prominent theory for why CPSP develops, termed the thermosensory disinhibition hypothesis, postulates that CPSP is a thermoregulatory disorder.[6,10] Specifically, damage to cooling-sensitive cortex in the dpIns (or to the cooling-sensitive (COOL) neurons in the lamina I STT that project via the lateral thalamus to this part of the dpIns) can lead to CPSP by disinhibiting 
activation of the ACC by polymodal nociceptive HPC (heat, pain, cold) lamina I STT neurons. These HPC neurons project to the ACC via the caudal part of the medial dorsal (MDvc) nucleus of the thalamus.

This is because temperatures below $25^{\circ} \mathrm{C}$ activate skin receptor species that input to both COOL and HPC lamina I STT neurons. In the normally functioning brain, at normal room temperatures, $\mathrm{COOL}$ input to the dpIns inhibits, via brainstem homeostatic sites, activation of the ACC by these HPC neurons. However, when the temperature falls to $15^{\circ} \mathrm{C}, \mathrm{COOL}$ activity plateaus, while HPC activity continues to rise. This leads to the ACC breaking free of inhibition and explains why thermal distress is experienced at temperatures below $15^{\circ} \mathrm{C} .[6,10]$ Thus, lesions which impair COOL activation of the dpIns can disinhibit the ACC. This is thought to lead to an imbalance in the bilateral integration of thermosensation and thermal distress at normal room temperatures. $[6,10]$

Ways of rebalancing this bilateral integration (e.g. "analgesic" contralateral strokes and vestibular stimulation) have been shown to alleviate CPSP. $[10,11]$ It is therefore noteworthy that the widespread neocortical and thalamic projections of the ZI include to the ipsilateral medial dorsal nucleus of thalamus and that the ZI also receives a dense projection from the ipsilateral ACC. $[12,13]$ As such, it is plausible that modulation of these structures by the ZI could be another means of rebalancing this integration.

Moreover, GABAergic projections from the ZI to the MDVc, inhibiting the HPC neurons there from activating the ACC could plausibly explain the neural mechanisms underpinning Lu et al.'s observations. As such, Figure 1 in Lu et al.'s report could be redrawn as shown here. It would be interesting to know what the study participants experienced phenomenologically. If they reported being aware of the sensation of the thermal pain, but without it distressing them, then this would lend weight to the interpretation of ACC inhibition being the mechanism. 
Third, the recent realization that GABAergic neurons in the ZI act to synchronize rather than simply inhibit cortical neurons has possible implications for the use of ventral ZI DBS in CPSP patients.[14,15] As Lu et al. state, the ZI is predominantly GABAergic and it does seem to be the case that ZI projections to thalamic relay nuclei can be involved in tonic inhibition.[15] However, it has become apparent in recent years that the action of the GABAergic neurons of the ZI is more nuanced than mere inhibition. Indeed, fast-firing ascending GABAergic ZI projections are associated with the synchronization of neuronal activity in various regions including the cortex and paradoxically the promotion of the type of fast EEG rhythms associated with arousal.[14,15] These fast-firing ventral ZI neurons discharge with a mean rate of $26 \mathrm{~Hz}$.[15] Thus, it is noteworthy that Lu et al. found that a "physiological" stimulation frequency of $20 \mathrm{~Hz}$ was more effective than $130 \mathrm{~Hz}$ in suppressing the perception of thermal pain. Whether this frequency is also most effective in alleviating the ongoing distress experienced by CPSP patients is, however, a question that will hopefully soon be answered empirically. 


\section{REFERENCES}

1. Lu, C.W., et al. Stimulation of zona incerta selectively modulates pain in humans. Sci. Rep. 11, 8924. https://doi.org/10.1038/s41598-021-87873-w (2021).

2. Dejerine, J. \& Roussy, G. The thalamic syndrome. In Rottenberg, D.A. \& Hochberg F.H. (Eds). Neurological classics in modern translation. New York: Hafner Press. p189-200 (1977).

3. Mulla, S.M. et al. Management of central poststroke pain. Stroke 46, 2853-2860. https://doi.org/10.1161/STROKEAHA.115.010259 (2015).

4. Gonzales, G.R. Suicide in central pain patients. Neurology 44 (Suppl 2): A318 (1994).

5. Craig, A.D. How do you feel--now? The anterior insula and human awareness. Nat. Rev. Neurosci. 10, 59-70. https://doi.org/10.1038/nrn2555 (2009).

6. Craig, A.D. (Bud). How Do You Feel? Princeton: Princeton University Press. (2015)

7. Rainville, P., Duncan, G.H., Price, D.D., Carrier, B. \& Bushnell, M.C. Pain affect encoded in human anterior cingulate but not somatosensory cortex. Science 277, 968-971. https://doi.org/10.1016/j.neuchi.2014.09.001 (1997).

8. Brooks, J.C., Nurmikko, T., Bimson, W.E., Singh, K.D. \& Roberts, N. fMRI of thermal pain: effects of stimulus laterality and attention. Neuroimage 15, 293-301. http://dx.doi.org/10.1006/nimg.2001.0974 (2002).

9. Iwata, K., et al. Anterior cingulate cortical neuronal activity during perception of noxious thermal stimuli in monkeys. J. Neurophysiol. 94, 1980-1991. https://doi.org/10.1152/jn.00190.2005 (2005).

10. Craig, A.D. Mechanisms of thalamic pain. In: Henry, J.L., Panju, A. \& Yashpal, K. (Eds). Central neuropathic pain: focus on poststroke pain. Seattle: IASP Press. p. 81-99 (2007).

11. McGeoch, P. D., Williams, L. E., Lee, R. R., \& Ramachandran, V. S. Behavioural evidence for vestibular stimulation as a treatment for central post-stroke pain. $J$. 
Neurol. Neurosurg. $\quad$ Psychiatry $\quad$ 79, 1298-1301. https://doi.org/10.1136/jnnp.2008.146738 (2008).

12. Merker, B. Consciousness without a cerebral cortex: a challenge for neuroscience and medicine. Behav. Brain Sci. 30, 63-134. https://doi.org/10.1017/S0140525X07000891 (2007).

13. Mitrofanis, J. Some certainty for the "zone of uncertainty"? Exploring the function of the zona incerta. Neuroscience 130, 1-15. https://doi.org/10.1016/j.neuroscience.2004.08.017 (2005).

14. Plaha, P., Khan, S., \& Gill, S. S. Bilateral stimulation of the caudal zona incerta nucleus for tremor control. J. Neurol. Neurosurg. Psychiatry 79, 504-513. https://doi.org/10.1136/jnnp.2006.112334 (2008).

15. Brown, R. E., \& McKenna, J. T. Turning a Negative into a Positive: Ascending GABAergic Control of Cortical Activation and Arousal. Front. Neurol. 6, 135. https://doi.org/10.3389/fneur.2015.00135 (2015). 
Acknowledgements

I thank Steven Gill for discussions and Chris Miskelly for modifying the figure.

Author Contribution Statement

PDM conceived and wrote the article himself.

Competing Interests Statement

None.

Figure Legend

Feedforward inhibition of thalamic pain processing by zona incerta. MDvc: ventrocaudal part of the medial dorsal nucleus. (Modified from Lu et al. 2021). 


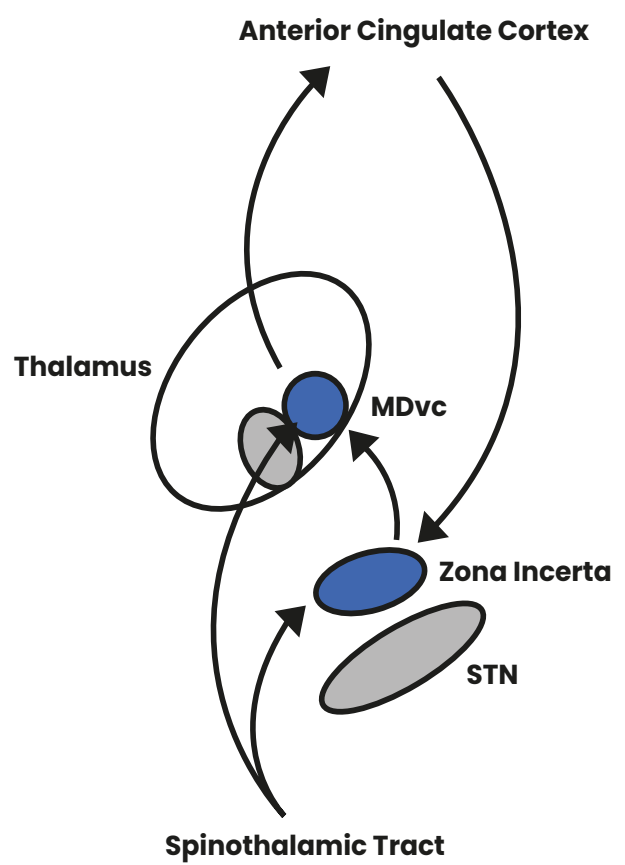

Figure 1. Feedforward inhibition of thalamic pain processing by zona incerta. MDvc ventrocaudal part of the medial dorsal nucleus. 